\title{
Surface Modification on Ultra High Molecular Weight Polyethylene Scaffold with Silk Fibroin
}

\author{
Jun Zhu, ${ }^{\ddagger, a}$ Jiejun Cheng, ${ }^{\ddagger, b}$ Juan Zhou, ${ }^{a}$ Gang Huang, ${ }^{*, c}$ and Dannong He $\mathrm{e}^{\star, a, b}$ \\ ${ }^{a}$ National Engineering Research Center for Nanotechnology, 28 East Jiangchuan Road, Shanghai 200241, \\ China \\ ${ }^{b}$ Department of Radiology, Shanghai Renji Hospital, Shanghai Jiao Tong University School of Medicine, \\ 1630 Dongfang Rd, Shanghai 200127, China \\ ${ }^{c}$ Shanghai Key Laboratory of Molecular Imaging, Shanghai University of Medicine \& Health Sciences, \\ 279 Zhouzhu Road, Shanghai 201318, China
}

Email: huang2802@163.com (G. H.), hdn_nercn@163.com (D. H.)

₹ These authors contributed equally to this work.

\begin{abstract}
In this paper, the surface of ultra high molecular weight polyethylene (UHMWPE) scaffold is modified to improve the hydrophilicity and cell adhesion behavior with silk fibroin (SF) grafting. Fourier Transform Infrared Spectroscopy (FT-IR), scanning electron microscope (SEM), Thermogravimetric (TG) and water contact angle analysis are performed to characterize the chemical structure, hydrophilicity and the morphology of UHMWPE/SF composites. The results show that SF can coat on the surface of UHMWPE scaffold, and the chromic acid etching is more conducive to SF deposition. After chrome-acid etching, both of the hydrophilicity and SF deposition on the surface of UHMWPE scaffold are improved, and the coating amount of SF is $10.31 \%$. Furthermore, there is no obvious decrease in mechanical properties, which proves that SF on the surface of UHMWPE scaffold has no effect on its mechanical properties. The cell adhesion experiments on UHMWPE scaffold are evaluated and the maximum cell viability appears in PECrSFs, which means that the biocompatibility is the best. All results have clearly indicated that the surface modification on UHMWPE scaffold with SF is an effective way of producing a suitably hydrophilic surface for their applications in biomedicine.
\end{abstract}

Keywords ultra high molecular weight polyethylene scaffold, silk fibroin, surface modification, biocompatibility

\section{Introduction}

Ultra high molecular weight polyethylene (UHMWPE) is used in medicine, mainly in the acetabulum and the sliding elements of endoprosthesis. Their advantages are primarily a very good tribological properties, toughness, durability, resistance to biological fluids and good mechanical properties. $^{[1,2]}$ Unfortunately, UHMWPE suffers from some drawbacks such as the low resistance to frictional wear a low yield strength and the tendency to creep and aging. One of the major disadvantages of UHMPE is their low surface energy, which makes them difficult to adhere to biological tissue. Therefore, UHMPE is often treated to improve their surface properties. For example, the surface modification improves the wear resistance of UHMWPE used in hip and knee implants. ${ }^{[3,4]}$ Free radicals, generated by ionizing radiation or chemically, react to form cross-links. ${ }^{[5,6]}$ Limiting cross-linking to the articulating surface of the implant is desirable to enable high wear resistance on the surface and higher strength and toughness in the bulk. ${ }^{[7]}$ Additionally, photochemical technique, plasma enhanced chemical vapor deposition (PCVD) and graft polymerization were also developed. ${ }^{[8-10]}$ However, these reports always focus on the mechanical properties of UHMWPE, ${ }^{[11,12]}$ and few research concentrate on the biocompatibility between and tissue.

Many implanted polymers, including polyethylene terephthalate (PET), polyetheretherketone (PEEK), polyvinylidene fluoride (PVDF), and so on, have been modified to improve their biocompatibility and bioactivity. Synthetic or natural polymers or inorganic material-based different scaffold formats have been used for maintaining cells in culture and tissue growth. Among them, silk fibroin (SF), a fibrillar protein, has attracted attention for support matrices for stem cells, nerve cells, fibroblasts, osteoblasts and other cell types, as well as scaffolds for many different types of tissues for engineering, including skin, nerve, bone, cartilage and blood vessels. ${ }^{[13-15]}$ Thus, SF has been used widely in surface modification of implanted polymers. For example, SF coating on PET via EDC/NHS crosslink could promote the compatibility of PET and enhance graft-bone healing anterior cruciate ligament $(A C L)$ reconstruction. ${ }^{[16]}$

In the present study, SF was performed onto the surface of UHMWPE scaffold to investigate the hydrophilic performance and biocompatibility of UHMWPE/SF composites. FT-IR, SEM, TG and water contact angle analysis are performed to characterize the chemical structure, hydrophilicity and the morphology of UHMWPE/SF composites. These results show that the sample surface modified with SF exhibited an improved hydrophilicity and bioactivity of cells.

\section{Experimental}

\section{Surface modification of UHMWPE scaffold with SF}

UHMWPE scaffold (recorded by PEs) with a size of $2 \mathrm{~cm} \times$ $2 \mathrm{~cm} \times 1 \mathrm{~mm}$ was soaked in ethanol with a concentration of $75 \%$ (VIV) for $2 \mathrm{~h}$. After being washed with deionized water to remove impurities from the surface, the sample was socked in chromic acid solution with $\mathrm{K}_{2} \mathrm{Cr}_{2} \mathrm{O}_{7}, \mathrm{H}_{2} \mathrm{O}$ and $\mathrm{H}_{2} \mathrm{SO}_{4}$ of $1: 2: 20$ $(w / w / w)$ with an ultrasonic treatment at $60{ }^{\circ} \mathrm{C}$ for $15 \mathrm{~min}$. The pretreated sample was obtained by washing with deionized water until aqueous solution is neutral and drying at the room 
temperature. The sample was recorded by PECrs

SF was prepared according to established procedures. ${ }^{[17]}$ Bombyx mori silk was degummed by means of boiling in $5 \mathrm{~g} / \mathrm{L}$ $\mathrm{Na}_{2} \mathrm{CO}_{3}$ solution for $1 \mathrm{~h}$. After sericin was removed, the degummed silk was dissolved in $\mathrm{CaCl}_{2} / \mathrm{H}_{2} \mathrm{O} / \mathrm{C}_{2} \mathrm{H}_{5} \mathrm{OH}$ ternary solvent for $40 \mathrm{~min}$ at $80^{\circ} \mathrm{C}$, specifically, $1 \mathrm{~g}$ degummed $\mathrm{SF}$ in $10 \mathrm{~mL}$ ternary solvent, followed by filtering and dialyzing against deionized water with a 14,000 molecular weight cut-off dialysis membrane for $72 \mathrm{~h}$ at room temperature. The dialyzed solution was collected that was $3 \%(w / w)$ aqueous regenerated SF solution. SF powder was prepared by casting the solution in freeze dryer at $80^{\circ} \mathrm{C}$.

The UHMWPE scaffold with chromium-treated was soaked in the $15 \% \mathrm{SF}$ solution, immersed for $12 \mathrm{~h}$ at $4{ }^{\circ} \mathrm{C}$ and vacuum-dried at room temperature. Furthermore, the sample was again soaked in $80 \%$ (VIV) ethanol solution for $2 \mathrm{~h}$ to fix the coating. Finally, the product was collected after drying at room temperature. The sample was recorded by PECrSFs.

Similarly, the control group of UHMWPE scaffold without treatment coating by SF was prepared according to the above method but free from the chromic acid treatment steps. The sample was recorded by PESFs.

\section{Characterization}

The morphologies of four groups (PEs, PECrs, PESFs and PECrSFs) were observed on field-emission scanning electron microscope (FESEM, Hitachi S-4800). The Fourier transformation infrared (FT-IR) spectra were recorded on a Nicolet 6700 FT-IR spectrometer. Thermogravimetric analyses (TGA) were conducted with a Linseis STA PT1600 thermoanalyzer instrument, with a temperature range of $25-650{ }^{\circ} \mathrm{C}$ at a heating rate of $10{ }^{\circ} \mathrm{C} / \mathrm{min}$ with $\mathrm{N}_{2}$ atmosphere. Contact Angle tester (JC2000C1) was used to determine the contact angle of the sample. The tensile properties of samples with $10 \mathrm{~cm} \times 0.6 \mathrm{~mm}$ in diameter were tested by CXT-4104 controlled by microcomputer with the tensile speed of the instrument was $10 \mathrm{~mm} / \mathrm{min}$.

\section{MTT assay}

NIH3T3 cells $\left(1 \times 10^{4}\right.$ cells per well) were seeded into 96-well flat-bottomed plates (Costar, Charlotte, NC, USA), and incubated with Dulbecco's Modified Eagle Medium (DMEM, Gibco, USA) supplied with $5 \% \mathrm{CO}_{2}$ at $37{ }^{\circ} \mathrm{C}$. After $24 \mathrm{~h}$, the cells were incubated with four groups (PEs, PECrs, PESFs and PECrSFs) in DMEM. Then, cells were treated with $5 \mathrm{mg} / \mathrm{mL}$ 3-(4,5-dimethylthiazol-2-yl)-2,5-diphenyltetrazolium bromide (MTT, $20 \mu \mathrm{L}$ in each well) in DMEM at $37{ }^{\circ} \mathrm{C}$ for $4 \mathrm{~h}$ for all samples. Formazan extraction was performed with DMSO, and then OD value (optical density) was measured at $492 \mathrm{~nm}$ by microplate reader. Six parallel replicates were read for each sample.

\section{Results and Discussion}

UHMWPE is chemically inactive, low surface energy, lack of polar groups, and a highly symmetrical methylene structure. The structure makes UHMWPE have high degree of crystallinity and orientation, and excellent mechanical properties. On the other hand, the chemical inertia of UHMWPE surface is particularly prominent, which leads to the poor biocompatibility of materials. Therefore, UHMWPE is not conducive to the adhesion, proliferation and differentiation of cells on the surface. In this study, UHMWPE scaffold is used as the matrix to improve their wet tability and biocompatibility by surface modification of SF. Meanwhile, to introduce a functional carboxyl group on the surface of UHMWPE, acid is utilized as previously reported, ${ }^{[18]}$ and the whole process is schematically illustrated in Figure 1.

Figure 2 shows FESEM images of PEs, PECrs, PESFs and

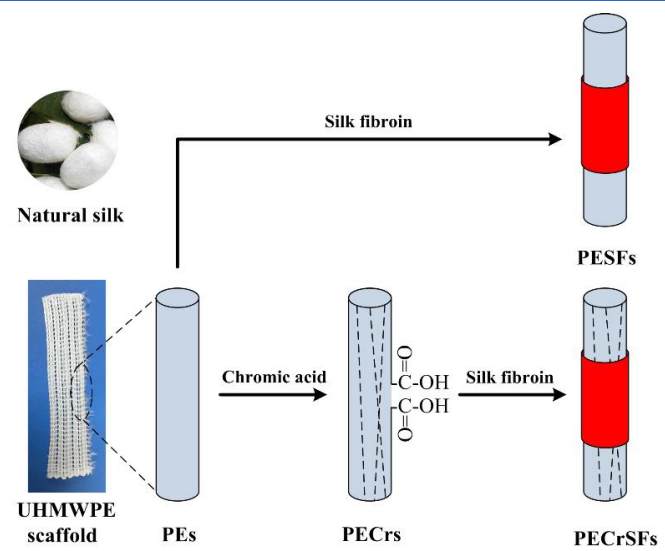

Figure 1 Schematic illustration of surface modification of SF on the surface of UHMWPE scaffold.

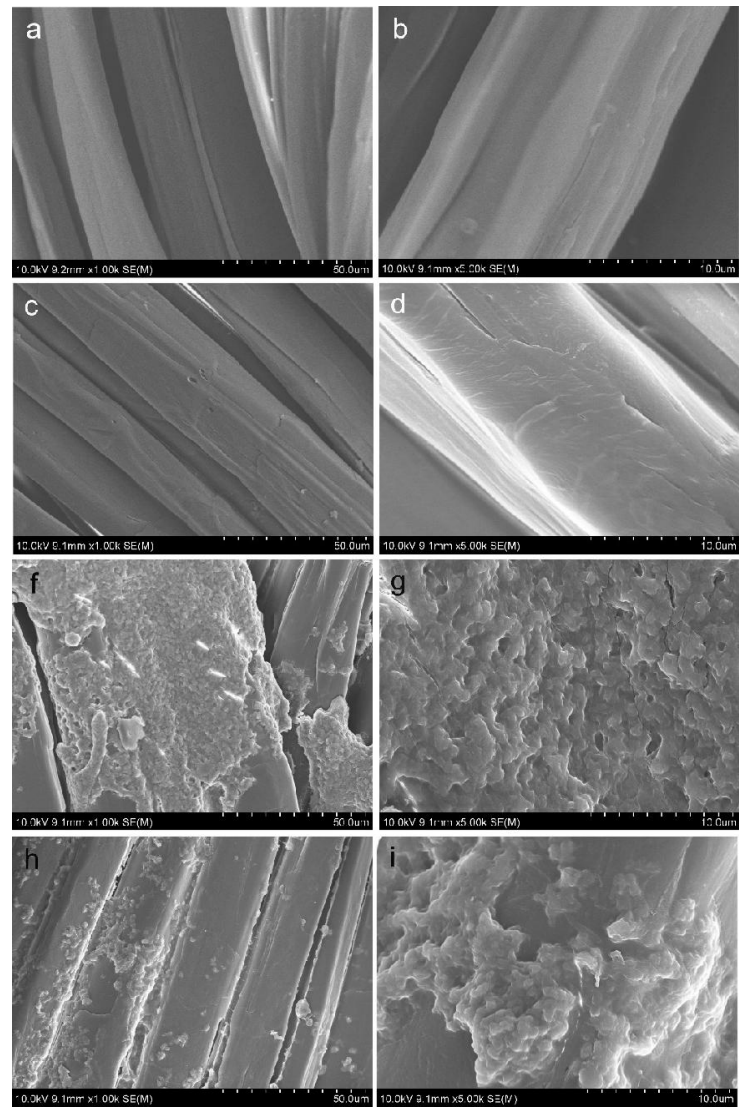

Figure 2 FESEM diagrams of (a, b) PEs, (c, d) PECrs, (f, g) PESFs and (h, i) PECrSFs.

PECrSFs. It can be seen that the fibers of UHMWPE scaffold with about $15 \mu \mathrm{m}$ in diameter are arranged in an orderly manner along a certain direction, and their surface is flat and smooth (Figures $2 a$ and $2 b$ ). After chromic acid etching, the fibers are still arranged regularly (Figure 1c), but their surface becomes wrinkled (Figure 2d). Furthermore, as the control group, PESFs without chromic acid treatment is covered with a layer of dense $\mathrm{SF}$, and their fiber diameter increased significantly (Figure 2f). It can be seen from the enlarged SEM image that there are a few cracks on the surface of SF coating (Figure $2 \mathrm{~g}$ ). Moreover, the surface of PECrSFs with chromic acid treatment is also covered with a dense SF coating (Figure 2h). There is no obvious interface between SF and the fiber surface of UHMWPE scaffold (Figure 2i). The result indicates that UHMWPE scaffold has a good interface binding effect with SF in PECrSFs, and the 
surface of PECrs material treated with chromic acid etching is more conducive to SF deposition.

TG curves of PEs, PECrs, PESFs and PECrSFs are shown in Figure 3. It can be seen that the curves of PEs and PECrs have the same trend, with only one weightlessness stage of broken chain degradation under $500{ }^{\circ} \mathrm{C}$. However, there are two weightlessness stages in both PESFs and PECrSFs curves. One stage is the decomposition of SF, which has an obvious weightlessness from 280 to $340^{\circ} \mathrm{C}$. The other stage contributes to the broken chain degradation from 340 to $500^{\circ} \mathrm{C}$. By comparing $\mathrm{P} 1$ and $\mathrm{P} 2$ sections in Figures $3 \mathrm{c}$ and $3 \mathrm{~d}$, it can be seen that the coating amount of SF coating in PESFs and PECrSFs is $8.66 \%$ and $10.31 \%$, respectively. After chrome-acid etching, the SF deposition on the surface of PEs is improved through enhancing PEs' mechanical locking for the interaction with SF.

a
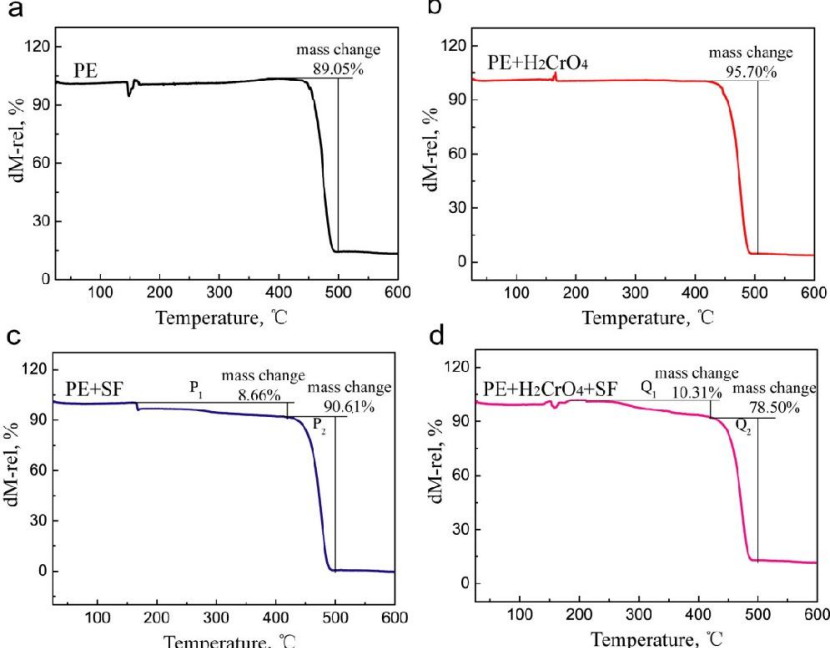

Figure 3 TG curves of (a) PEs, (b) PECrs, (c) PESFs and (d) PECrSFs.

Figure 4A shows the FT-IR spectrum for PEs, PECrs, PESFs, and PECrSFs. It can be seen that the absorption peaks of PEs at 719, 729, 1463, 2850 and $2913 \mathrm{~cm}^{-1}$ are characteristic peaks of $\mathrm{C}-\mathrm{H}$ bond of PEs in curve a. Furthermore, $\mathrm{PECrs}$ treated with chromic acid etching have the same characteristic peaks as PEs, meaning their position and strength does not change significantly. The result indicates that the chemical structure of PEs has not been changed by chromic acid treatment. Moreover, as shown in curves $c$ and $d$ in Figure 4A, PESFs and PECrSFs shows the absorption peaks at 1629 and $1520 \mathrm{~cm}^{-1}$, which attributes to the characteristic peaks of silk protein with beta-folded structure of amide I belt and amide II belt, respectively, while the characteristic peaks from PEs are significantly weakened or disappeared. The result implies that SF coating is deposited on the surface of PEs, according to
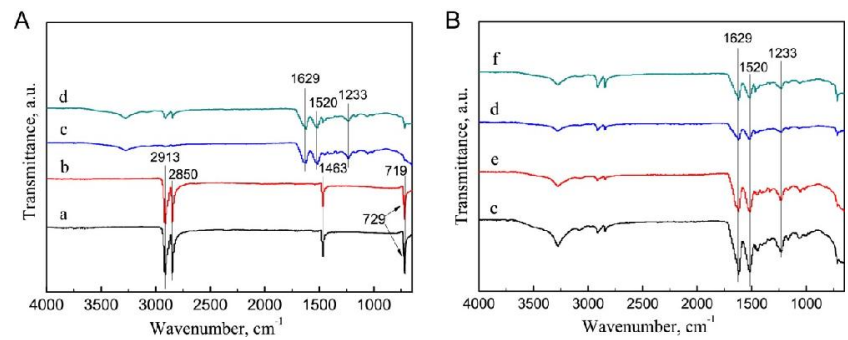

Figure 4 (A) FT-IR spectrum of (a) PEs, (b) PECrs, (c) PESFs and (d) PECrSFs and (B) FT-IR spectrum of (c) PESFs, (d) PECrSFs and their immersion in water for 24 hours for (e) PESFs and (f) PECrSFs.
SEM results of Figure 2. In order to further study the solubility of coating, PESFs and PECrSFs were soaked in water for $24 \mathrm{~h}$. As shown in Figure 4B, it can be seen that the dissolution or disappearance of soluble silk protein coating is not obvious compared by curves $c$ and $e$ and curves $d$ and $f$, which indicates PEs has a good surface binding effect with SF.

The method of static contact angle was used to evaluate the hydrophilicity of the samples throughout the experiments. ${ }^{[19]}$ Figure 5 shows the contact angles of PEs, PECrs, PESFs, and PECrSFs with water. Because the surface of PEs lacks polar groups and is a typical hydrophobic material, its contact angle with water is $92^{\circ} \mathrm{C}$ (Figure 5a). However, as shown in Figure 5b, the contact angle between PECrs and water decreased to $66.5{ }^{\circ} \mathrm{C}$ after chromic acid treatment, meaning that the hydrophilicity of PECrs is significantly improved (Figure $5 \mathrm{~b}$ ). The reason can result from $P E s$ surface being roughened under chromic acid etching, which increases the specific surface area and surface energy of the fiber. Moreover, as shown in Figures $5 \mathrm{c}$ and $5 \mathrm{~d}$, the contact angles of PESFs and PECrSFs are 77 and $56{ }^{\circ} \mathrm{C}$, respectively. The minimum water contact angle of $56{ }^{\circ} \mathrm{C}$ appears in PECrSFs, which means that the hydrophilicity is the best. In other word, a strongest interface binding between SF and UHMWPE scaffold lies in PECrSFs, which is according to Figure 2. The results imply that SF coating further improves the hydrophilicity of PEs and provides a better surface environment for cell adhesion and proliferation.
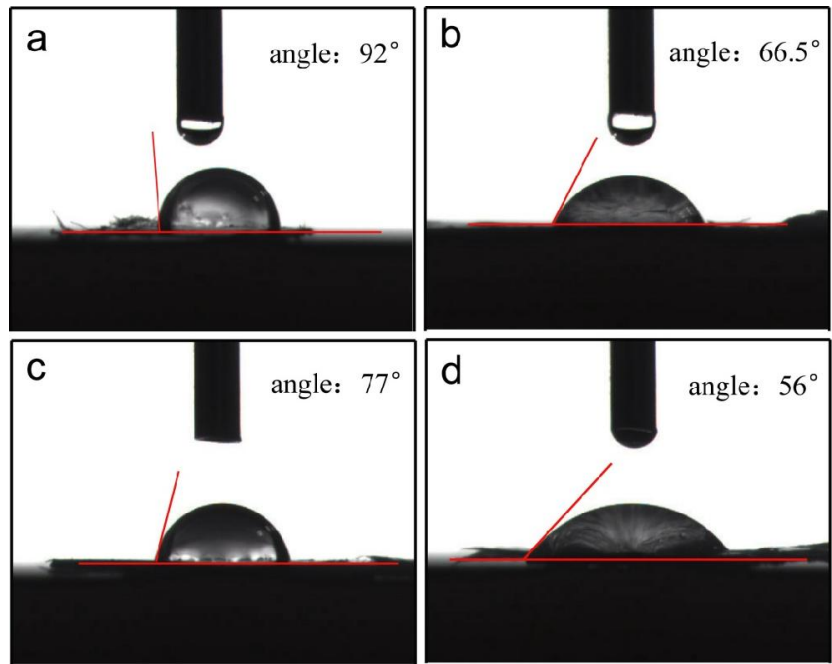

Figure 5 Contact angle images of (a) PEs; (b) PECrs; (c) PESFs and (d) PECrSFs.

As one of the high performance reinforced materials, UHMWPE has been widely used due to its unique mechanical properties of high strength and modulus, excellent corrosion and wear resistance. Therefore, mechanical performance was evaluated by tensile strength. Figure 6 shows the tensile properties of PEs, PECrs, PESFs and PECrSFs. The tensile strengths of PEs, PECrs, PESFs and PECrSFs are $1.676 \pm$ $0.041,1.609 \pm 0.053,1.629 \pm 0.037$ and $1.488 \pm 0.062 \mathrm{GPa}$, respectively. The result indicates that there is no obvious decrease in mechanical properties, which proves that SF on the surface of UHMWPE scaffold has no effect on its mechanical properties.

To examine the biocompatibility and bioactivity of the PEs, PECrs, PESFs and PECrSFs for the application in biomedicine, their cytotoxicity was investigated by MTT assay. As shown in Figure 7, the effect of different groups on cells proliferation was assessed with NIH3T3 cells. Using the viability of untreated cells as control, the cellular viabilities change with different 


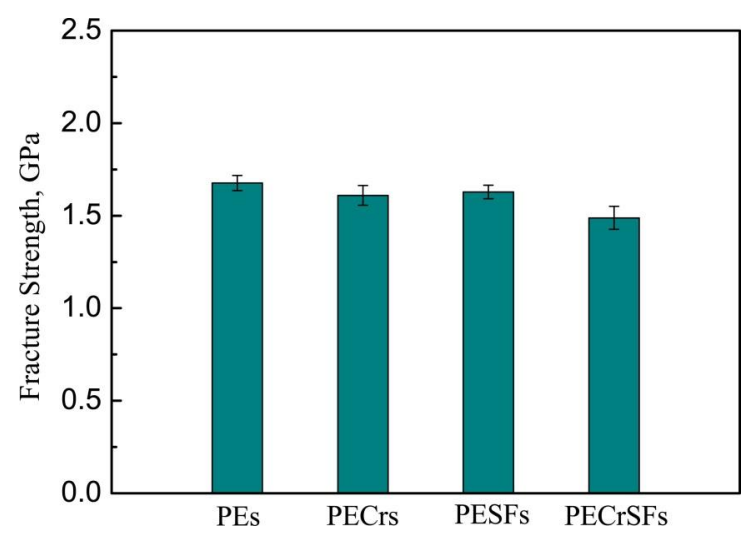

Figure 6 Tensile performance of PEs, PECrs, PESFs and PECrSFs.

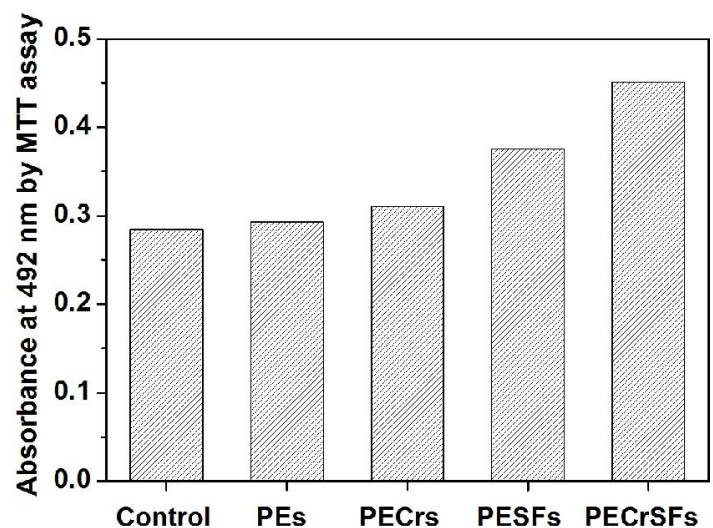

Figure 7 The OD value of PEs, PECrs, PESFs and PECrSFs group at 1 days $(n=7)$.

treatment manners after $1 \mathrm{~d}$ of induction. The number of cells on the PESFs and PECrSFs is significantly greater than that on the PEs and PECrs, which means that the high hydrophilicity would be conducive to the cell adhesion and spreading. ${ }^{[20]}$ Specially, the maximum OD value appears in PECrSFs, which means that its biocompatibility is the best.

\section{Conclusions}

UHMWPE scaffold is modified by SF. Both of the hydrophilicity and SF deposition on the surface of UHMWPE scaffold is improved, and the coating amount of SF is $10.31 \%$. Furthermore, there is no obvious decrease in mechanical properties, which proves that SF on the surface of UHMWPE scaffold has no effect on its mechanical properties. The cell adhesion experiments on UHMWPE scaffold are evaluated and the maximum cell viability appears in PECrSFs, which means that the biocompatibility is the best. All results have clearly indicated that the surface modification on UHMWPE scaffold with SF is an effective way of producing a suitably hydrophilic surface for their application in biomedicine.

\section{Acknowledgement}

The work was supported by the Shanghai Science and Technology Development Fund (Nos. 17XD1421900 and 17441902700), the Shanghai Shenkang Project (No. 16CR3024A) and the Shanghai Key Laboratory of Molecular Imaging (No. 18DZ2260400).

\section{References}

[1] Firdous, S.; Fuzail, M.; Atif, M.; Nawaz, M. Optik 2011, 122, 99.

[2] Bladen, C. L.; Teramura, S.; Russell, S. L.; Fujiwara, K.; Fisher, J.; Ingham, E.; Tomita, N.; Tipper, J. L. J. Biomed. Mater. Res. B Appl. Biomater. 2013, 101, 458

[3] Shanbhag, A. S.; Bailey, H. O.; Hwang, D. S.; Cha, C. W.; Eror, N. G.; Rubash, H. E. J. Biomed. Mater. Res. 2000, 53, 100.

[4] Kurtz, S. M.; Muratoglu, O. K.; Evans, M.; Edidind, A. A. Biomaterials 1999, 20, 1659.

[5] Oral, E.; Beckos, C. G.; Malhi, A. S.; Muratoglu, O. K. Biomaterials 2008, 29, 3557.

[6] Slouf, M.; Kotek, J.; Baldrian, J.; Kovarova, J.; Fencl, J.; Bouda, T.; Janigova, I. J. Biomed. Mater. Res. B Appl. Biomater. 2013, 101 , 414.

[7] Kayandan, S.; Doshi, BN.; Oral, E.; Muratoglu, OK. J. Biomed. Mater. Res. B Appl. Biomater. 2018, 106, 1517.

[8] Chu, Y. Y.; Chen, X. G.; Tian, C. L. Appl. Surf. Sci. 2017, 406, 77.

[9] Mori, M.; Uyama, Y.; Ikada, Y. J. Polym. Sci. Pol. Chem. 1994, 32, 1683.

[10] Kavc, T.; Kern, W.; Ebel, M. F.; Svagera, R.; Pölt, P. Chem. Mater. 2000, 124, 1053

[11] Turell, M. B.; Bellare, A. Biomaterials 2004, 25, 3389.

[12] Grygiel, M.; Kaczmarek, M. IEEE Trans. Inf. Technol. Biomed. 2014, 4, 395.

[13] Altman, G. H.; Diaz, F.; Jakuba, C.; Calabro, T.; Horan, R. L.; Lu, H.; Richmond, J.; Kaplan, D. L. Biomaterials 2003, 24, 401.

[14] Wang, Y.; Bella, E.; Lee, C. S. D.; Migliaresi, C.; Pelcastre, L.; Schwartz, Z.; Boyan, B. D. ; Motta, A. Biomaterials 2010, 31, 4672.

[15] Partlow, B. P.; Hanna, C. W.; Rnjak-Kovacina, J.; Moreau, J. E.; Applegate, M. B.; Burke, K. A.; Marelli, B.; Mitropoulos, A. N.; Omenetto, F. G.; Kaplan, D. L. Adv. Funct. Mater. 2014, 24, 4615.

[16] Ai, C. C.; Cai, J. Y.; Zhu, J.; Zhou, J.; Jiang, J.; Chen, S. Y. RSC Adv. 2017, 7, 51303.

[17] Zhou, J.; Zhang, B.; Shi, L.; Zhong, J.; Zhu, J.; Yan, J.; Wang, P. Cao, C. B.; He, D. N. ACS Appl. Mater. Interfaces 2014, 6, 21813.

[18] Fávaro, S. L.; Rubira, A. F.; Muniz, E. C.; Radovanovic, E. Polym. Degrad. Stab. 2007, 92, 1219.

[19] Zheng, Z. W.; Ren, Li.; Feng, W. J.; Zhai, Z. C.; Wang, Y. J. Appl. Surf. Sci. 2012, 258. 7207.

[20] Ishihara, K.; Iwasaki, Y.; Ebihara, S.; Shindo, Y.; Nakabayashi, N. Colloids Surf. B Biointerfaces 2000, 18, 325

Received June 12, 2019 Accepted July 7, 2019 